\title{
The roles of miRNA, IncRNA and circRNA in the development of osteoporosis
}

\author{
Yang Yang ${ }^{2 \dagger}$, Wang Yujiao ${ }^{3+}$, Wang Fang ${ }^{1}$, Yuan Linhui ${ }^{3}$, Guo Ziqi $^{3}$, Wei Zhichen ${ }^{3}$, Wang Zirui ${ }^{3}$ \\ and Wang Shengwang ${ }^{1 *}$
}

\begin{abstract}
Osteoporosis is a common metabolic bone disease, influenced by genetic and environmental factors, that increases bone fragility and fracture risk and, therefore, has a serious adverse effect on the quality of life of patients. However, epigenetic mechanisms involved in the development of osteoporosis remain unclear. There is accumulating evidence that epigenetic modifications may represent mechanisms underlying the links of genetic and environmental factors with increased risk of osteoporosis and bone fracture. Some RNAs, such as microRNAs (miRNAs), long noncoding RNAs (IncRNAs), and circular RNAs (circRNAs), have been shown to be epigenetic regulators with significant involvement in the control of gene expression, affecting multiple biological processes, including bone metabolism. This review summarizes the results of recent studies on the mechanisms of miRNA-, IncRNA-, and circRNA-mediated osteoporosis associated with osteoblasts and osteoclasts. Deeper insights into the roles of these three classes of RNA in osteoporosis could provide unique opportunities for developing novel diagnostic and therapeutic approaches to this disease.
\end{abstract}

Keywords: Osteoporosis, MiRNA, Lncrna, CircRNA

\section{Background}

Human bone metabolism is a complex process including bone absorption and bone formation mediated by osteoblasts and osteoclasts. Osteoporosis, a metabolic skeletal disease caused by decreased bone formation and increased bone absorption, is characterized by reduced bone mass and deterioration of the microstructure of the bone tissue, and is associated with increased fracture risk [1] (Fig. 1). Previous studies have shown that the occurrence of osteoporosis is related to many factors, including genetic and environmental factors. Most of these factors influence the development of osteoporosis by interfering with osteoblast and osteoclast differentiation and activity. As a widespread and complex disease, the incidence

\footnotetext{
*Correspondence: 1256821502@qq.com

†Yang Yang and Wang Yujiao contributed to this work equally and should be regarded as co-first authors

1 People's Hospital of Guazhou County, Guazhou 736100, Gansu, China

Full list of author information is available at the end of the article
}

of osteoporosis is increasing markedly with the aging of the population [2]. Osteoporosis is a chronic disease that has adverse effects on quality of life, morbidity, and even mortality. It is estimated that more than 75 million people have osteoporosis worldwide [3]. At present, the approved treatments for osteoporosis mainly include selective estrogen receptor modulators, bisphosphonates, denosumab, and teriparatide, etc. But these methods have limited efficacy as well as numerous adverse effects, and there is as yet no highly effective treatment for osteoporosis $[4,5]$. This disease is a major global health problem that places a heavy burden on patients and society. However, recent studies of epigenetics have provided insights that may lead to the possibility of osteoporosis treatment.

Epigenetics is the regulation of gene expression without changes to the underlying DNA sequence [6], and includes DNA methylation, histone modifications, and RNA-based mechanisms [7]. These modifications are expected to result in variable expression of identical 


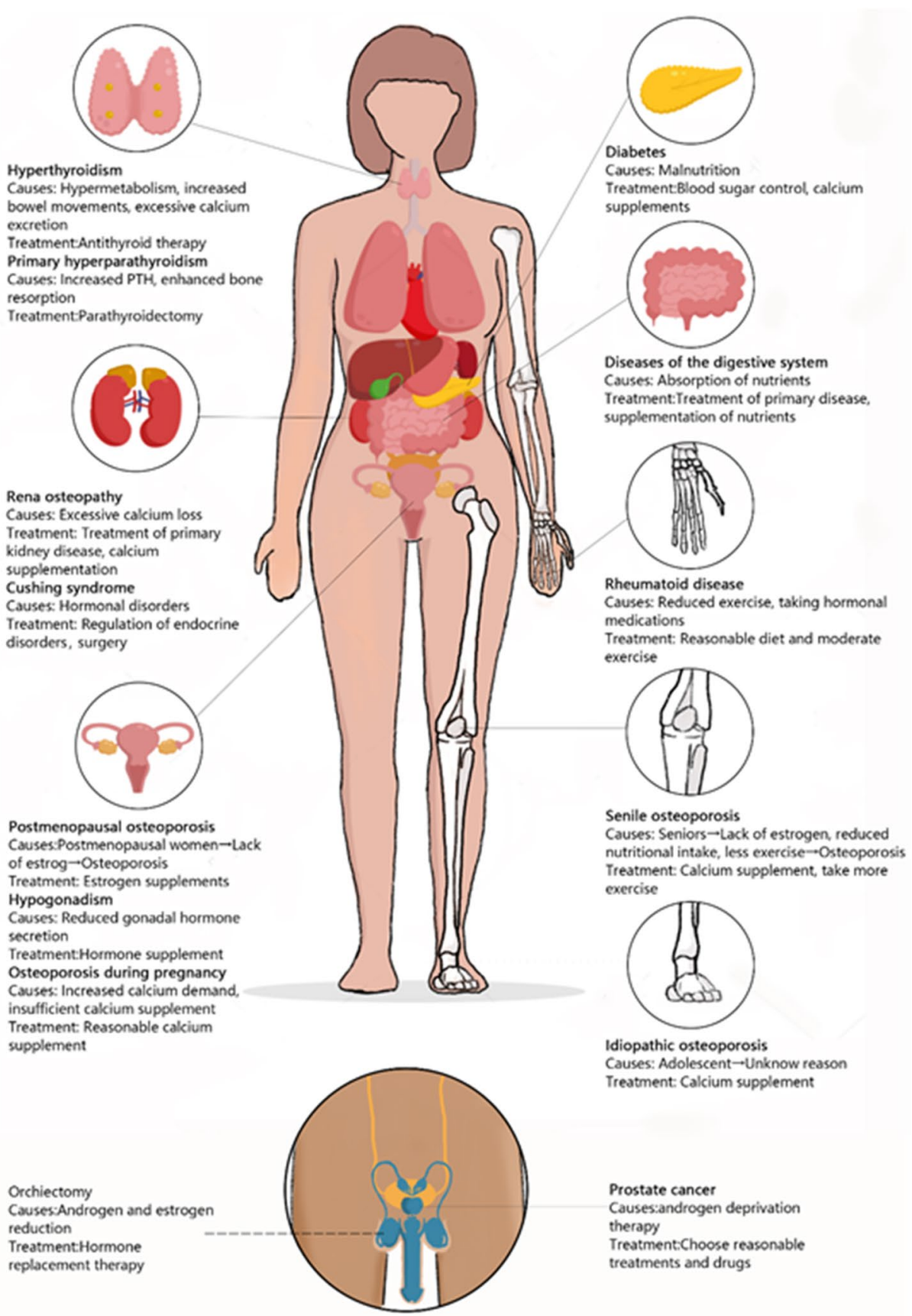

Fig. 1 Main causes and diseases of osteoporosis. Main causes of osteoporosis development

genetic information based on the surrounding conditions leading to enhanced expression or silencing of genes [8]. With the in-depth study of epigenetics, there is accumulating evidence that microRNAs (miRNAs), long noncoding RNAs (lncRNAs), and circular RNAs (circRNAs) are closely related to the occurrence and development of various diseases, including inflammatory diseases, metabolic diseases, and cancer $[9,10]$. One study had shown that 13 miRNAs, 70 lncRNAs and 260 circRNAs were differentially expressed in patients with postmenopausal osteoporosis (OP group) compared with healthy controls (NC group) [11]. As a multifactorial disease, osteoporosis is closely related to epigenetics. As potential therapeutic targets or biomarkers, there is increasing 
research and clinical interest in miRNAs, lncRNAs, and circRNAs. The main goal of this review was to set out the roles of miRNAs, lncRNAs, and circRNAs in the occurrence and development of osteoporosis, as related to their effects on osteoblasts and osteoclasts, to provide a theoretical basis for exploring the pathogenesis and potential for clinical treatment of the disease (Fig. 2).

\section{miRNAs, IncRNAs, circRNAs}

\section{miRNAs}

The miRNAs are small $(\sim 22$ nucleotides $)$, singlestranded, non-coding RNAs encoded by endogenous genes, which have important regulatory effects in cells. miRNAs bind to complementary sequences in their target mRNAs, thereby suppressing expression of the target mRNAs through posttranscriptional regulation, forming a complex regulatory network $[12,13]$.

It has been speculated that miRNAs regulate one-third of human genes and participate in a number of vital life processes [14].

miRNA-based gene therapy is a rapidly developing disease treatment strategy with a number of advantages. Such therapeutic strategies have great potential for the treatment of osteoporosis.

\section{IncRNAs}

The lncRNAs, which are large non-coding RNAs $>200$ nucleotides in length, play important roles in various activities of life [15], including dose compensation effects, epigenetic regulation, and regulation of cell differentiation. Abnormalities of IncRNAs could cause disease, and many studies have shown that ncRNAs are closely related to the mechanism underlying the pathogenesis of osteoporosis.

\section{circRNAs}

The circRNAs, first identified in the cytoplasm of mammalian cells, are more durable than linear RNAs as they have a stable loop structure that prevents exonucleasemediated degradation [16]. It has been demonstrated circRNAs possess abundant miRNA binding sites, and can act as miRNA sponges. Thus, circRNAs play an essential regulatory role in disease through interactions with disease-related miRNAs [17].

\section{Mechanisms of action of these three types of RNAs} in regulation of osteoblast differentiation in osteoporosis Osteoblasts are mainly differentiated from mesenchymal progenitor cells in the inner and outer periosteum and bone marrow. These cells specifically secrete a

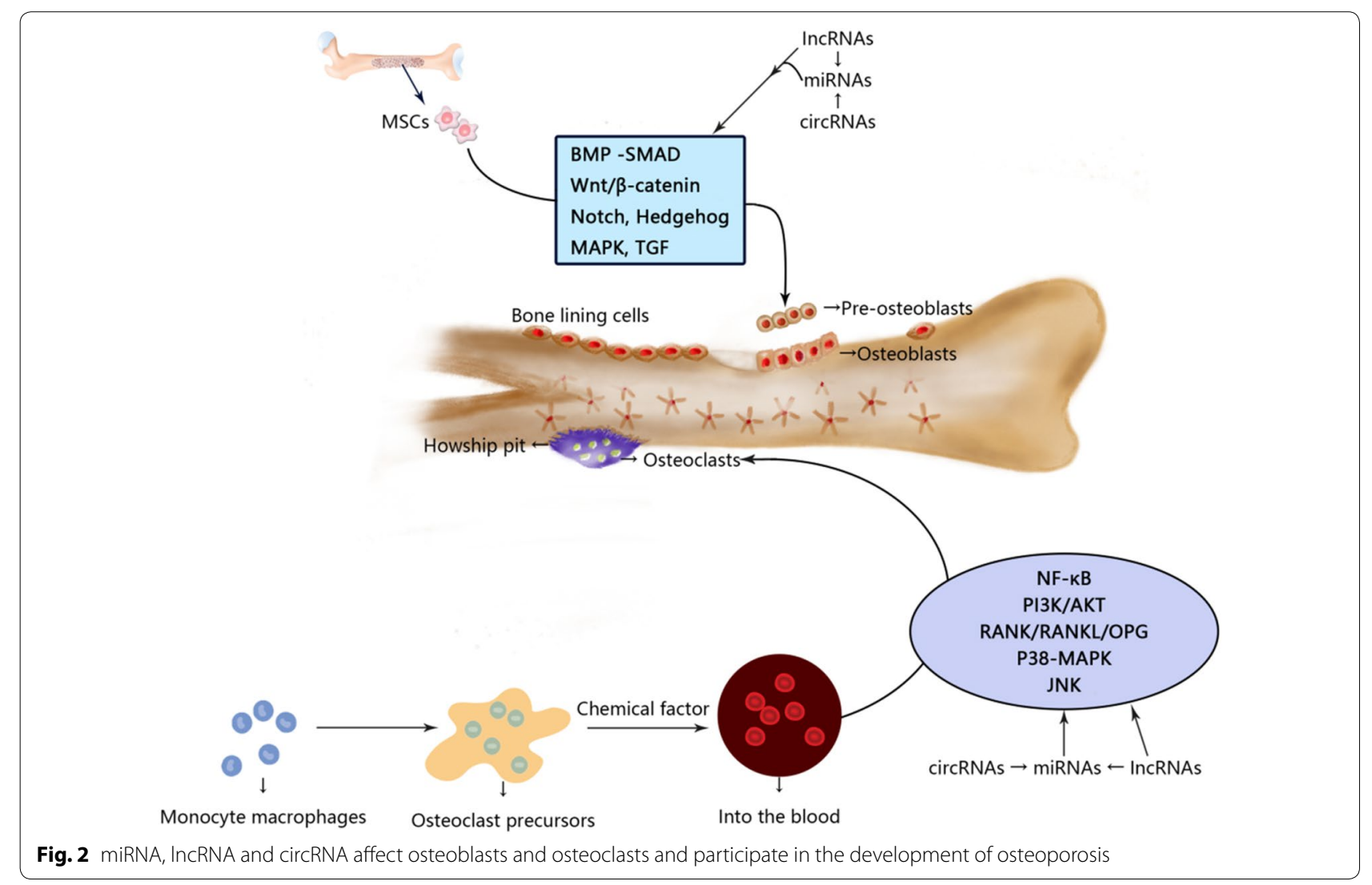


variety of biologically active substances. In bone tissue, a number of signaling pathways are involved in the regulation of bone homeostasis by promoting bone formation [18]. For example, after stimulating the Wnt/ $\beta$-catenin signaling pathway, glycogen synthase 3 activity decreased and $\beta$-catenin phosphorylation and proteasome degradation were inhibited. Therefore, phosphorylated $\beta$-catenin collected in the cytoplasm and were then translocated to the nucleus to deactivate gene transcription. This induced the proliferation and differentiation of osteoblasts and reduced apoptosis of mature osteoblasts [19]. As the foremost functional cells for bone formation and reconstruction, osteoblasts are responsible for the synthesis, secretion, and mineralization of bone matrix. Therefore, the differentiation and activity of osteoblasts are particularly closely connected to the occurrence and development of osteoporosis. Here, we review the mechanisms by which miRNAs, lncRNAs, and circRNAs mediate osteoblast differentiation. Adjusting these related molecules may be helpful in the treatment of osteoporosis (Fig. 3).

\section{miRNA-mediated mechanisms}

Some miRNAs have been shown to promote the differentiation of osteoblasts in osteoporosis by regulating the substances related to their differentiation. These miRNAs have an inhibitory effect on the progression of osteoporosis. For example, miR-33-5p acts as a mechanosensitive microRNA that positively regulates osteoblastogenesis by repressing high mobility group AT-hook 2 (Hmga2), which is an inhibitor of osteoblastogenesis [20]. As a key transcription factor, Runt-related transcription factor 2 (Runx2) acts as a modulator regulating the differentiation of mesenchymal stem cells (MSCs) into osteoblasts, which further mature into osteocytes [21]. miRNA-194 promotes osteoblast differentiation by regulating Runx2 nuclear translocation mediated by signal transducer and activator of transcription (STAT1) [22]. As an important biomarker of osteoporosis, Dickkopf-1 (DKK1) is an antagonist of the WNT signaling pathway, and elevation of its level is related to osteolytic bone disease. miRNA-433-3p inhibits the expression of DKK1 protein, activates the $\mathrm{Wnt} / \beta$-catenin signaling pathway, and promotes osteoblast differentiation [23]. Some groups have suggested that miR-96 inhibits the phosphorylation of epidermal growth factor receptor (EGFR), extracellular

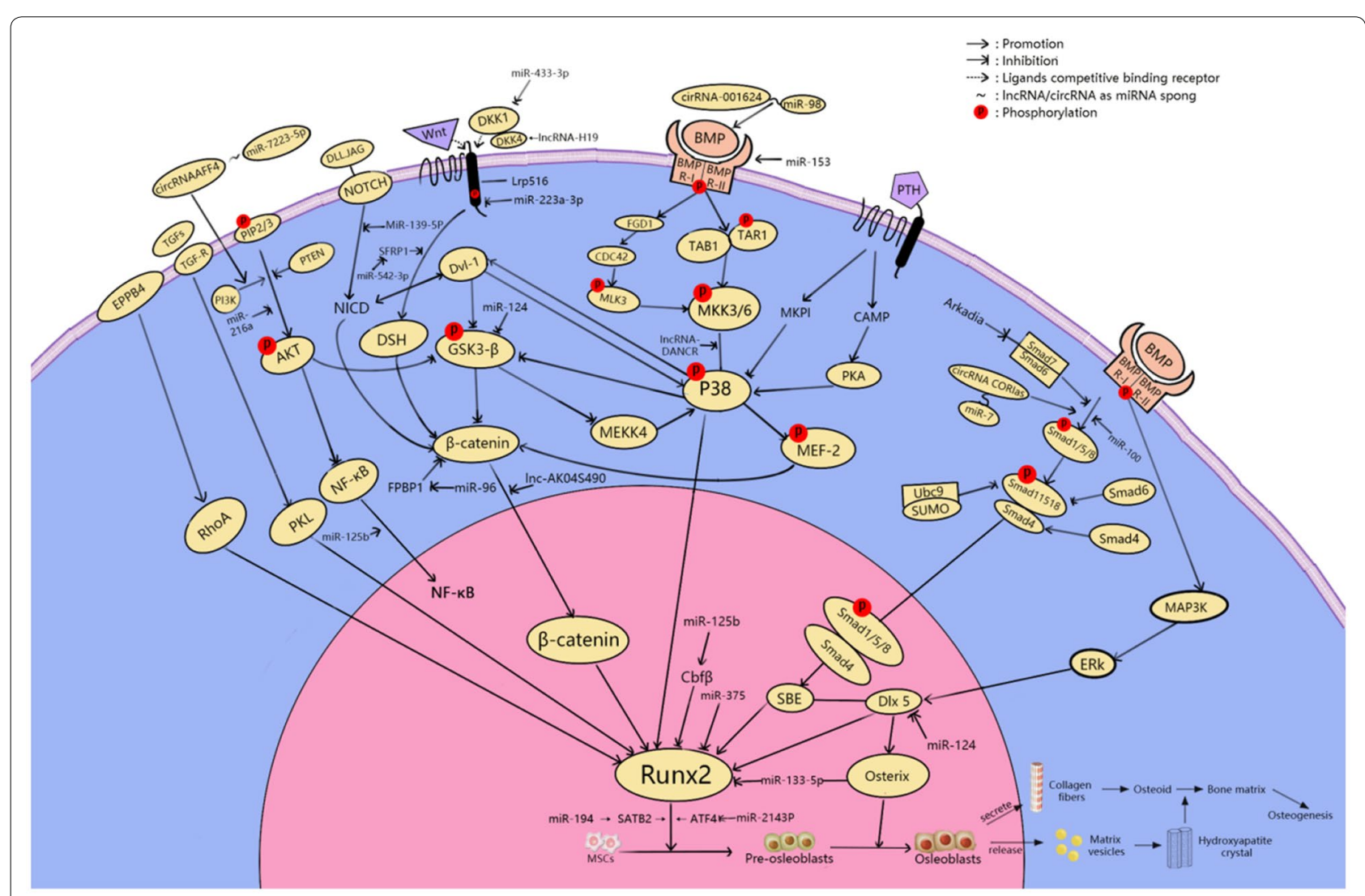

Fig. 3 Schematic diagram of the mechanism of miRNA, IncRNA and circRNA affecting osteoblasts in osteoporosis 
signal-regulated kinase 1 (ERK1), and AKT through the downregulation of Human Proheparin-Binding EGF-like Growth Factor (HB-EGF) induced by binding to the $3^{\prime}$ untranslated region (UTR) of its mRNA, thus promoting osteogenic differentiation [24]. In bone tissue, canonical Wnt-signaling is a major regulator of bone homeostasis by promoting bone formation [25]. Another experimental study suggested that miR-96 can activate the Wnt signaling pathway to promote osteogenic differentiation. Animal experiments showed that the level of alkaline phosphatase (ALP) activity, number of calcium nodules, and osteoblast viability increased when the expression level of miR-96 was increased [26]. Besides miR-96, the downregulation of microRNA-139-5p was also reported to positively regulate the $\mathrm{Wnt} / \beta$-catenin signaling pathway by inducing Notch1 signaling and eventually affecting osteogenic differentiation [27]. The $\mathrm{PI} 3 \mathrm{~K} / \mathrm{AKT}$ pathway is another important pathway in bone metabolism, and miR-216a was shown to regulate the c-Cbl-mediated PI3K/AKT pathway, thereby preserving osteogenesis and enhancing osteoblast differentiation and bone formation [28].

Besides the promoting effects of several miRNAs outlined above, overexpression of some miRNAs could also inhibit osteoblast differentiation and make healing of osteoporosis(it refers to the reduction of symptoms and the increase of BMD) difficult. miR-125b inhibits osteogenic differentiation. On the one hand, it was shown to attenuate osteoblast differentiation of periodontal ligament cells (PDLCs) by targeting NKIRAS2 and enhancing NF- $k B$ signaling [29]. On the other hand, miR-125b was reported to regulate the osteogenic differentiation of human bone marrow-derived mesenchymal stem cells (hBMSCs) by targeting BMPR1b [30]. In the early stages of osteoblast differentiation, miR-125b acts indirectly on Runx 2 by targeting a putative binding site for the $3^{\prime}$-UTR of the Cbf $\beta$ gene, a key transcription factor for osteogenesis, thereby inhibiting its differentiation [31]. Besides miR-125b, both miR-375 and miRNA-133a-5p could target Runx2. However, miR-375 suppresses osteogenic differentiation by directly targeting Runx 2 and, in this process, it reduces the activity levels of key osteoblast markers, including osteocalcin, ALP, and collagen type I $\alpha 1$ (COL1A1) [32]. miRNA-133a-5p inhibits the expression of Runx2, at both mRNA and protein levels by targeting the $3^{\prime}$-UTR of Runx2 [33]. As an endogenous attenuator of Smad1, miR-100 was shown to inhibit BMP-induced osteoblast differentiation [34]. Similarly, miR-203a-3p was reported to suppress osteoblast differentiation by regulating smad9, and the Wnt3a/ $/$-catenin signaling pathway was activated following miR-203a-3 inhibition [35]. Transfer of osteoclast-derived exosomal miR-214-3p to osteoblasts inhibited bone formation [36], which was mediated by inhibition of the protein expression of an important osteogenic transcription factor, ATF4, a target of miR-214-3p in osteoblasts [37]. miR153 , a mechanosensitive miRNA, was shown to regulate osteoblast differentiation by directly targeting BMPR2 [38].

Furthermore, miR-124 and miR-542-3p showed dualdirectional regulation of osteoblast differentiation. Their roles in osteoporosis would differ depending on the specific physiopathological state. First, miR-124 was shown to promote the proliferation and differentiation of osteoblasts via the BMP/TGF- $\beta$ signaling pathway [39]. Moreover, miR-124 was reported to directly target the $3^{\prime}$-UTRs of Dlx3, Dlx5, and Dlx2, which are negative regulators of osteogenic differentiation and bone formation in vivo [40]. Downregulation of miR-124 expression was reported to enhance the expression of GSK-3 $\beta$, a serine/threonine kinase that can regulate cell differentiation, and then attenuate the activity of the Wnt $/ \beta$-catenin signaling pathway inhibiting differentiation of osteoblasts [41]. miR-542-3p shows a dual role in osteogenic differentiation. On the one hand, miR-542-3p was shown to play an important positive role in bone formation by inhibiting expression of the secreted frizzled-related protein-1 (SFRP1), a negative regulator of the WNT signaling pathway, and to induce osteoblast differentiation [42]. On the other hand, overexpression of miR-542-3p caused repression of BMP-7 and inhibition of BMP-7/PI3K survival signaling, which would limit osteogenic differentiation and promote osteoblast apoptosis [43].

Based on the above findings, some groups have proposed that comparing miRNA levels in serum before and after osteoporosis treatment would further verify their mechanism of action in mediating osteoporosis. After 3 months of treatment with TBTD, miR-33 expression was downregulated, and serum levels of miR-133a were reduced after 12 months of treatment. Although miR-124 level did not change significantly with TPTD treatment, the level of miR-124 expression was lower after the third month of treatment and the responsiveness of bone mineral density (BMD) to TPTD treatment was higher at the twelfth month [44]. Other studies showed that teriparatide and zoledronate could regulate miRNA levels in the treatment of osteoporosis. For example, they increased the expression of miR-203a-3p in the tissues and serum of untreated ovariectomized animals [45]. In summary, miRNAs are closely related to osteoblast differentiation. Therefore, these specific miRNAs may be useful therapeutic targets for targeted medication, and it may be possible to treat osteoporosis by adjusting their content and expression levels in patients. Furthermore, miRNAs transported through vesicles can serve as diagnostic markers in osteoporosis treatment (Table 1). 
Table 1 The role of some miRNAs in osteoporosis and their mechanisms through osteoblasts and osteoclasts and targets

\begin{tabular}{|c|c|c|}
\hline miRNA ID & Target molecule and pathway & $\begin{array}{l}\text { Mechanisms that affect osteoporosis } \\
\text { through osteoblasts and osteoclasts }\end{array}$ \\
\hline miR-33-5p [20] & Hmga2 & Promote osteoblast differentiation \\
\hline miR-194 [22] & Runx2 & Promote osteoblast differentiation \\
\hline miRNA-433-3p [23] & DKK1 & Promote osteoblast differentiation \\
\hline $\operatorname{miR}-96[24,26]$ & $\begin{array}{l}\text { EGFR, HB-EGF } \\
\text { Wnt/ß-catenin signaling pathway }\end{array}$ & $\begin{array}{l}\text { Promote osteoblast differentiation } \\
\text { Promote osteoblast differentiation }\end{array}$ \\
\hline miR-139-5p [27] & $\mathrm{NOTCH} 1, \mathrm{Wnt} / \beta$-catenin pathway & Promote osteoblast differentiation \\
\hline miR-216a [28] & $\begin{array}{l}\text { PI3K/AKT pathway } \\
\text { BMP/TGF- } \beta \text { signaling pathway, }\end{array}$ & $\begin{array}{l}\text { Promote osteoblast differentiation } \\
\text { Promote osteoblast differentiation }\end{array}$ \\
\hline miR-124 [39-41] & $\begin{array}{l}\text { GSK-3 } \beta \text {, Wnt/ } \beta \text {-catenin pathway } \\
\text { Dlx3, Dlx5, and Dlx2 }\end{array}$ & $\begin{array}{l}\text { Inhibit osteoblast differentiation } \\
\text { Inhibit osteoblast differentiation }\end{array}$ \\
\hline miR-542-3p $[42,43]$ & $\begin{array}{l}\text { SFRP1 } \\
\text { BMP-7/PI3K- survivin signaling } \\
\text { NKIRAS2, NF-KB signaling }\end{array}$ & $\begin{array}{l}\text { Promote osteoblast differentiation } \\
\text { Inhibit osteoblast differentiation } \\
\text { Inhibit osteoblast differentiation }\end{array}$ \\
\hline miR-125b [29-31] & BMPR1b & Inhibit osteoblast differentiation \\
\hline miR-375 [32] & Runx2 & Inhibit osteoblast differentiation \\
\hline miR-100 [34] & Smad1 & Inhibit osteoblast differentiation \\
\hline miR-203a-3p [35] & Smad9, Wnt/ß-catenin signaling pathway & Inhibit osteoblast differentiation \\
\hline $\operatorname{miR}-214-3 p[35,37]$ & ATF4 & Inhibit osteoblast differentiation \\
\hline miR-153 [38] & BMPP2 & Inhibit osteoblast differentiation \\
\hline $\operatorname{miR}-21[69,70]$ & RANKL, PI3K/Akt signaling pathway, PDCD4 & Promote osteoclast differentiation \\
\hline miR-183 [71] & RANKL, HO-1 & Promote osteoclast differentiation \\
\hline miR-155 $[72,73]$ & $\begin{array}{l}\text { TNF- } \alpha \text {, IL-1 } \beta \text {, RANKL, M-CSF, RANK, TRAP, BCl-2, LEPR, AMPK, } \\
\text { p-AMPK, OPG, Bax, TAB } 1\end{array}$ & Promote osteoclast differentiation \\
\hline miR-223 [74] & TWIST and Runx2 & Promote osteoclast differentiation \\
\hline miR-19a [74] & TWIST and Runx2 & Promote osteoclast differentiation \\
\hline miR-214 [75] & Pten, PI3K/Akt pathway & Promote osteoclast differentiation \\
\hline miR-182 [76] & Foxo3, Maml1 & Promote osteoclast differentiation \\
\hline miR-26a [77] & CTGF/CCN2 & Inhibit osteoclast differentiation \\
\hline miR-31 [78] & RhoA & Inhibit osteoclast differentiation \\
\hline miRNA-17 [79] & RANKL & Inhibit osteoclast differentiation \\
\hline miR-503 [80] & RANK & Inhibit osteoclast differentiation \\
\hline miR-126-5p $[81,82]$ & PTHrP and MMP-13 & Inhibit osteoclast differentiation \\
\hline miR-7b [83] & DC-STAMP & Inhibit osteoclast differentiation \\
\hline miR-141 [84] & Calcr, EphA2 & Inhibit osteoclast differentiation \\
\hline
\end{tabular}

\section{IncRNA-mediated mechanisms}

A number of lncRNAs have been shown to promote osteoblast differentiation, and it was speculated that they may be useful in the treatment of osteoporosis. First, the histone decarboxylase SIRT1 was shown to be an important positive regulator of osteoblastogenesis and bone mass. However, the expression of SIRT1 was inversely proportional to the expression of lncRNA HIF1A-AS1, suggesting the function of IncRNA HIF1A-AS1 in osteogenic differentiation, although the specific mechanism is not yet clear [46]. Second, lncRNA HoxA-AS3 was reported to interact with Enhancer Of Zeste 2 (EZH2) and to be required for $\mathrm{H} 3$ lysine-27 trimethylation (H3K27me3) of the key osteogenic transcription factor Runx2. Therefore, lncRNA HoxA-AS3 is a significant molecule in osteoblast differentiation [47].

In contrast to lncRNA HoxA-AS3, lncRNA-DANCR was shown to recruit enhancer of zeste homolog 2 (EZH2) to promote H3K27me3 by interacting with 305-nt transcript and enhancer of zestehomolog2, ultimately inhibiting transcription of the target gene Runx2 and osteogenic differentiation [48]. Moreover, DANCR also mediated the proliferation and osteogenic differentiation of hBMSCs through inactivation of p38 MAPK [49]. IncRNA ANCR was shown to be a fundamental regulator of osteogenic differentiation; when 
upregulated, it inhibited osteogenic differentiation by inhibiting signaling pathways [50]. Furthermore, lncRNAs that inhibited osteoblasts in differentiation through the $\mathrm{WNT} / \beta$-catenin signaling pathway included lncRNA HOTAIR and lncRNA AK045490. IncRNA HOTAIR downregulated the expression of $\mathrm{Wnt} / \mathrm{\beta}$-catenin signaling pathway-related proteins to inhibit signal transduction and inhibited osteoblast differentiation. DKK1 was when to reduce the protein levels of HOTAIR, $\beta$-catenin, Cyclin D, C-myc, and Runx2, which could partially reverse the regulatory effect of HOTAIR on Wnt/ $\beta$ catenin [51]. Moreover, when the expression level of lncRNA p21 is decreased, the Wnt/ $\beta$-catenin signaling pathway is activated by increased secretion of E2, finally stimulating bone formation and osteogenic differentiation [52]. The DKK4 gene encodes a protein belonging to the Dickkopf family. Downregulation of lncRNA H19 reduced the expression level of Dkk4, thereby inhibiting the $\mathrm{Wnt} / \beta$-catenin signaling pathway and negatively regulating osteogenic differentiation [53]. lnc-AK045490, which is enriched in skeletal tissues, inhibited osteoblast differentiation and bone formation by inhibiting nuclear translocation of $\beta$-catenin and downregulating the expression of TCF1, LEF1, and Runx2 [54]. Similarly, lncAK016739 inhibited osteogenic differentiation and bone formation because it could inhibit the expression and activity of osteoblastic transcription factors [55]. Inhibition of IncRNA UCA1 was shown to promote osteoblast proliferation and differentiation by activating the BMP-2/ (Smad1/5/8) signaling pathway in osteoblasts [56].

Furthermore, lncRNA MEG3 plays dual roles in the differentiation of osteoblasts, showing both positive and negative regulation. First, lncRNA MEG3 was shown to promote the proliferation and differentiation of osteoblasts by activating the Wnt/ $\beta$-catenin signaling pathway, so it is expected to become a new target for accelerating fracture healing [57]. However, downregulation of lncRNA MEG3 inhibited osteogenic differentiation by enhancing expression of IGF1 [58]. The observations outlined above suggest that most lncRNAs have inhibitory effects against osteogenic differentiation. Therefore, it may be possible to silence the expression of these specific lncRNAs using specifically targeted drugs, thereby limiting the development of osteoporosis (Table 2).

\section{Circular RNA-mediated mechanisms}

circRNAs have also been reported to play roles in osteoblast differentiation and thus also participate in the development of osteoporosis. During osteogenesis, circRNA_0016624 was reported to activate miR-98 and enhance BMP2 expression, which is known to play an important role in induction of osteogenic differentiation, and so circRNA_0016624 promoted osteoblast differentiation [59]. Acting as a sponge for miR-7223-5p, circRNA AFF4 promoted MC3T3-E1 cell proliferation and inhibited apoptosis under the action of PI3KR1 [60]. circRNA CDR1as was shown to be an inhibitor of miR7, which upregulated GDF5 expression, and stimulated Smad1/5/8 and p38 MAPK phosphorylation, thereby promoting osteogenic differentiation of PDLSCs [61]. Studies have shown that circHIPK3 expression was reduced in necrotic bone tissue, and miR-124 was upregulated after targeted knockout of circHIPK3, thereby increasing osteoblast cell death. Dexamethasone- and $\mathrm{H}_{2} \mathrm{O}_{2}$-induced cytotoxicity in osteoblasts was/were reported to be associated with downregulation of $\mathrm{circH}$ IPK3 [62, 63]. In contrast, an inverse correlation was observed between circIGSF11 and miR-199b-5p. It found that this correlation was upregulated during the osteogenesis of hBMSCs. Silencing of circIGSF11 promoted osteoblast differentiation and increased the expression of miR-199b-5p [64]. At present, only the mechanisms of action of the above-mentioned circRNAs in osteoblast differentiation have been determined. There has been less research on circRNAs compared with miRNAs and IncRNAs, and this should be the subject of future studies related to osteoporosis (Table 3).

\section{Mechanisms of action three kinds of RNA in regulation of osteoclast differentiation in osteoporosis}

Osteoclasts, derived from blood mononuclear macrophages, are terminally differentiated cells. The principal function of osteoclasts is to mediate bone resorption, and they play an important role in bone development, growth, repair, and reconstruction. Abnormal osteoclast function can cause abnormal bone resorption. Hyperfunction can cause bone degenerative diseases, such as osteoporosis, so osteoclast differentiation plays a significant role in osteoporosis. RANK was osteocalcin receptor of RANKL. RANK recognizes and binds with RANKL on the surface of osteoclasts and osteoclast progenitor cells in a cell type-dependent manner, and has been show to directly promote the differentiation, activation, and maturation of osteoclasts. As a signaling molecule, RANKL effectively induces the generation and differentiation of osteoclasts. Osteoprotegerin (OPG) is a new member of the tumor necrosis factor (TNF) receptor family, known as osteoclastogenesis inhibitory factor (OCIF), so it has the function of inhibiting osteoclasts [65-67]. Therefore, the RANK/RANKL/OPG pathway is very important in bone tissue metabolism [68].

As mentioned above, anomalous increases in osteoclast number would accelerate the formation and progression of osteoporosis. Previous studies have shown that miRNAs, lncRNAs, and circRNAs affect osteoclast differentiation, and so they also have roles in osteoporosis. Here, 
Table 2 The role of some IncRNAs in osteoporosis and their mechanisms through osteoblasts and osteoclasts and targets

\begin{tabular}{|c|c|c|}
\hline LncRNA ID & Target molecule and pathway & Effects on osteoblasts differentiation \\
\hline IncRNA HIF1A-AS1 [46] & SIRT1 & Promote osteoblast differentiation \\
\hline LncRNA HoxA-AS3 [47] & $\mathrm{EZH} 2, \mathrm{H} 3 \mathrm{~K} 27 \mathrm{me} 3$, Runx2 & Promote osteoblast differentiation \\
\hline IncRNA MALAT1 [102] & miR-143, miR-204 & Promote osteoblast differentiation \\
\hline IncRNA MODR [103] & miR-454 & Promote osteoblast differentiation \\
\hline LncRNA KCNQ1OT1 [104] & miR-214 & Promote osteoblast differentiation \\
\hline LncRNA NTF3-5 [105] & miR-93-3p & Promote osteoblast differentiation \\
\hline LncRNA POIR [106] & miR-182 & Promote osteoblast differentiation \\
\hline LncRNA Linc-ROR [107] & miR-145 & Promote osteoblast differentiation \\
\hline LncRNA H19 $[100,101]$ & miR-675, miR-141, miR-22 & Promote osteoblast differentiation \\
\hline LncRNA H19 [53] & Wnt/ß-catenin pathway, & Inhibit osteoblast differentiation \\
\hline LncRNA-DANCR $[48,49]$ & EZH2, H3K27me3, Runx2, p38 MAPK & Inhibit osteoblast differentiation \\
\hline LncRNA ANCR [50] & Wnt/ß-catenin pathway & Inhibit osteoblast differentiation \\
\hline LncRNA HOTAIR [51] & Wnt/ $\beta$-catenin pathway & Inhibit osteoblast differentiation \\
\hline IncRNA p21 [52] & E2, Wnt/ß-catenin pathway & Inhibit osteoblast differentiation \\
\hline Lnc-AK045490 [54] & $\beta$-catenin, TCF1, LEF1 and Runx2 & Inhibit osteoblast differentiation \\
\hline Lnc-AK016739 [55] & osteoblastic TF & Inhibit osteoblast differentiation \\
\hline IncRNA UCA1 [56] & BMP-2/(Smad1//5/8) & Inhibit osteoblast differentiation \\
\hline \multirow[t]{2}{*}{ LncRNA MEG3 $[57,58]$} & Wnt/ß-catenin signaling pathway & Promote osteoblast differentiation \\
\hline & IGF1 & Inhibit osteoblast differentiation \\
\hline LncRNA HOTAIR [108] & miR-17-5p, SMAD7 & Inhibit osteoblast differentiation \\
\hline LncRNA MIAT [109] & miR-150-5p & Inhibit osteoblast differentiation \\
\hline IncRNA-ORLNC1 [111] & miR-296 & Inhibit osteoblast differentiation \\
\hline LncRNA MEG3 [112] & miR-133a-3p & Inhibit osteoblast differentiation \\
\hline LncRNA TSIX [52] & miR-30a-5p, and Runx2 & Promote osteoblast apoptosis \\
\hline IncRNA TUG1 [86] & PTEN & Promote osteoclast differentiation \\
\hline IncRNA AK077216 [87] & NIP45, NFATc1 & Promote osteoclast differentiation \\
\hline IncRNA SNHG15 [88] & RANK/RANKL pathyway & Promote osteoclast differentiation \\
\hline LncRNA-Jak3 [89] & Nfatc1, Ctsk & Promote osteoclast differentiation \\
\hline LncRNA LINC00311 [90] & DDL3 & Promote osteoclast differentiation \\
\hline LncRNA RP11-498C9.17 [91] & HDAC4 & Inhibit osteoclast differentiation \\
\hline LncRNA Bmncr [92] & RANK & Inhibit osteoclast differentiation \\
\hline LncRNA NONMMUT037835.2 [93] & RANK, NF-KB/MAPK signaling pathway & Inhibit osteoclast differentiation \\
\hline LncRNA-NEF [94] & $\mathrm{IL}-6$ & Inhibit osteoclast differentiation \\
\hline
\end{tabular}

we summarized these relevant research results to provide new ideas and a foundation for research and treatment of osteoporosis from the perspective of osteoclasts (Fig. 4).

\section{microRNA-mediated mechanisms}

Here, we discuss miRNAs that could promote the process of osteoclast differentiation. In theory, these miRNAs may have adverse effects on osteoporosis. Previous studies had shown that multiple miRNAs could affect differentiation of osteoclasts induced by RANKL. First, during osteoclastogenesis, miR-21 could be upregulated by RANKL and may promote osteoclastogenesis and bone resorption by targeting Pten to activate the PI3K/ Akt signaling pathway or targeted downregulation of programmed cell death protein 4 (PDCD4) $[69,70]$. Second, increased RANKL-induced miR-183 can inhibit heme oxygenase-1 (HO-1) by binding to its $3^{\prime}$-UTR, thereby positively regulating osteoclastogenesis [71]. Third, downregulation of miR-155 can decrease TNF- $\alpha$, IL-1 $\beta$, RANKL, M-CSF, RANK, TRAP, and Bcl-2 expression, and increase LEPR, AMPK, p-AMPK, OPG, and Bax expression, thus inhibiting osteoclast proliferation and bone resorption [72]. Lipopolysaccharide (LPS)induced miR-155 promoted autophagy to increase osteoclast formation by decreasing Table 2 expression [73]. Fourth, miR-223 and miR-19a regulated the expression of Twist and Runx2, affected the expression of RANK/ RANKL pathway and MCP-1, and ultimately regulated 


\begin{tabular}{|c|c|c|}
\hline circRNA ID & Target molecule and pathway & $\begin{array}{l}\text { Mechanisms that affect osteoporosis } \\
\text { through osteoblasts and osteoclasts }\end{array}$ \\
\hline circRNA_0016624 [59] & miR-98, BMP2 & Promote osteoblast differentiation \\
\hline circRNA AFF4 [60] & miR-7223-5p & Promote osteoblast differentiation \\
\hline CircRNA CDR1as [61] & miR-7, GDF5, Smad1/5/8 and p38 MAPK & Promote osteoblast differentiation \\
\hline circHIPK3 $[62,63]$ & miR-124 & Promote osteoblast differentiation \\
\hline circRNA436 [1 111$]$ & miR-108, miR-335 & Promote osteoblast differentiation \\
\hline CircRNA BANP [112] & miRNA-146a, PDGFRA & Promote osteoblast proliferation \\
\hline circRNA ITCH [112] & miR-34a, DUSP1 & Promote osteoblast proliferation \\
\hline circlGSF11 [64] & miR-199b-5p & Inhibit osteoblast differentiation \\
\hline hsa_circ_0127781 [111] & miR-210, miR-335 & Inhibit osteoblast differentiation \\
\hline CircRNA_28313 [95] & miR-195a, CSF1 & Promote osteoclast differentiation \\
\hline CircRNA_007438 [96] & miRNA-6338, miRNA-7028-3p & Promote osteoclast differentiation \\
\hline circRNA_005108 [96] & $\begin{array}{l}\text { miRNA-6975-3p, miRNA-6516-5p, miRNA-486b-5p, miRNA- } \\
31-3 p\end{array}$ & Inhibit osteoclast differentiation \\
\hline
\end{tabular}

the pathophysiology of osteolytic bone destruction [74]. Fifth, as a key regulator of skeletal metabolism, on the one hand, miR-214 promoted osteoclast activity by targeting Pten via the PI3K/Akt pathway. On the other hand, it also mediated osteoclast-osteoblast crosstalk through exocrine miRNA paracrine mechanisms [75]. Finally, miR-182 promoted TNF- $\alpha$-induced osteoclastogenesis by inhibiting Foxo3 and Maml1, and this process was controlled by RBP-J [76]. The above findings suggested that drugs may affect the development and treatment of osteoporosis by affecting the expression of the abovementioned miRNAs. In contrast, the miRNAs discussed below can inhibit osteoclast differentiation by targeting related molecules, and so may allow the development of innovative methods for treating osteoporosis. RANKL was shown to upregulate the expression of miR-26a at the late stage of osteoclastogenesis. A number of miR-26a inhibitors can enhance RANKL-induced osteoclast formation and CTGF expression. Connective tissue growth factor/CCN family 2 (CTGF/CCN2) was shown to promote osteoclast formation by suppressing the expression of dendritic cell-specific transmembrane protein (DC-STAMP). Its expression could be downregulated by miR-26a mimic in osteoclast precursor cells resulting in the inhibition of osteoclast formation, actin ring formation, and bone resorption [77]. Second, miR-31 was shown to inhibit RANKL-induced osteoclast formation by regulating RhoA expression (RhoA plays an important role in the regulation of cytoskeleton remodeling) [78]. Third, miR-17/20a targeting reduces the expression of RANKL in osteoclasts; thus, it has an inhibitory effect on osteoclast differentiation [79]. Finally, RANK is the target of miR-503, which can bind to RANKL and induce osteoclast differentiation and activation, and silencing of miR-503 in CD14 ${ }^{+}$PBMCs promotes osteoclastogenesis [80]. The RANK/RANKL/OPG pathway is closely connected with osteoclasts, but miRNA can also regulate osteoclast differentiation via other mechanisms. miR-126-5p is an intronic miRNA that can control osteoclast differentiation by negatively regulating PTHrP and MMP-13 [81, 82]. miR-7b was reported to inhibit osteoclastogenesis and cell fusion by directly targeting DC-STAMP [83]. miR-141 has been identified as a quintessential negative inhibitor of osteoclastogenesis and bone resorption. The functional mechanism involves its targeting of two osteoclast differentiation factors, calcitonin receptor (Calcr) and ephrin A receptor 2 precursor (EphA2) [84].

Recent experiments have shown that oleanolic acid, a new drug that can be used to treat osteoporosis, inhibits RANKL-induced osteoclastogenesis through the ER $\alpha$ / miR-503/RANK signaling pathway in RAW264.7 cells [85]. This represents an example of successful osteoporosis treatment based on miRNA. With the exception of miR503, the above studies demonstrated that these miRNAs are important signaling molecules in the RANK/ RANKL/OPG pathway and may serve as therapeutic targets for the prevention and treatment of osteoporosis (Table 1).

\section{IncRNA-mediated mechanisms}

lncRNAs have been shown to regulate osteoclastogenesis by regulating the expression of specific target mRNAs. In osteoporosis, the increased expression of lncRNA TUG1 can regulate the proliferation and apoptosis of osteoclasts through Pten [86]. During the formation of osteoclasts, 


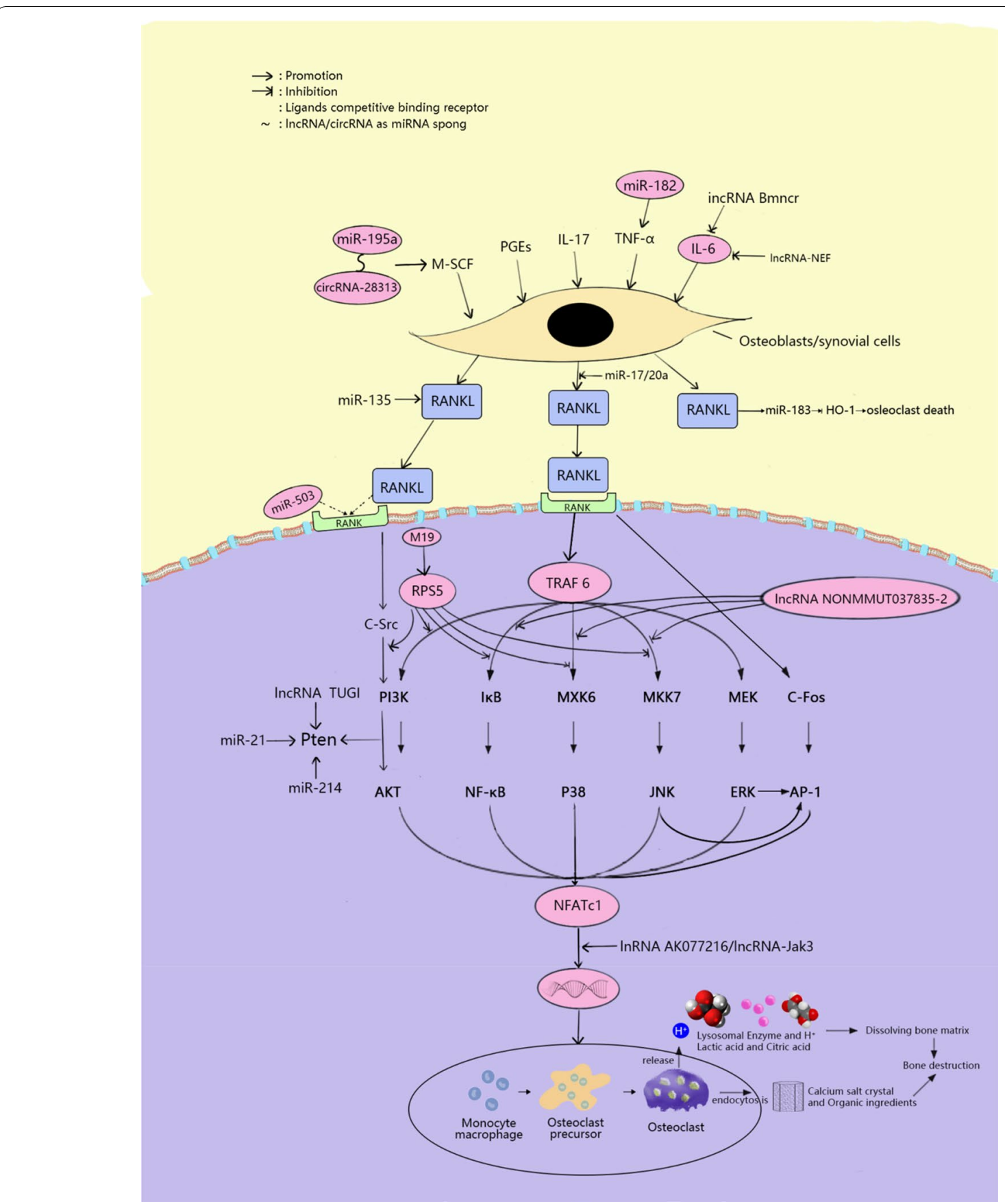

Fig. 4 Schematic diagram of the mechanism of miRNA, IncRNA and circRNA affecting osteoclasts in osteoporosis

the expression of lncRNA AK077216 was significantly up-regulated. This lncRNA inhibits the expression of NIP45 and promotes the expression of NFATc1, a major transcription factor involved in osteoclast differentiation [87]. Downregulation of lncRNA SNHG15 expression suppressed osteoclasts by regulating the RANK/RANKL pathway [88]. IncRNA-Jak3-mediated Nfatc1 activation was reported to upregulate cathepsin K (Ctsk) expression to promote MSU-induced osteoclast differentiation [89]. lncRNA LINC00311 promoted osteoclast differentiation by inhibition of DDL3 expression and regulating the Notch signaling pathway [90].

Of course as expected, lncRNAs also have inhibitory effects on osteoclasts. The expression levels of these 
lncRNAs were inversely associated with the severity of osteoporosis. IncRNA RP11-498C9.17 was closely related to various epigenetic regulatory factors, such as HDAC4, MORF4L1, HMGA1, and DND1. Among them, downregulation of histone deacetylase was shown to promote osteoclast differentiation, suggesting that lncRNA RP11498C9.17 may regulate osteoclast production through HDAC4 [91]. IncRNA Bmncr is also a negative regulator of RANKL-induced osteoclast differentiation [92]. IL-6 intervened in regulation of bone mineral density and osteoclast differentiation and activation. IncRNA-NEF may play a role in osteoporosis by inhibiting IL-6. Studies in postmenopausal osteoporosis showed that a high lncRNA-NEF level was associated with a substantially reduced course of treatment and lower post-treatment recurrence rate [93]. IncRNA NONMMUT037835.2 regulated osteoclastogenesis by negatively regulating RANK expression and inhibiting the NF-KB/MAPK signaling pathway. Upregulation of lncRNA NONMMUT037835.2 inhibited osteoclast differentiation [94].

In summary, these lncRNAs that had effects on osteoclast differentiation may represent a breakthrough in the treatment of osteoporosis. Decreases and increases in the lncRNA expression levels promoted and suppressed osteoclast differentiation, respectively (Table 2 ).

\section{circRNA-mediated mechanisms}

So far, most studies had focused on miRNAs, and there were few studies on the relationship between circRNAs and osteoclasts. Among them, the research on circRNA_28313 was relatively clear. It relieved miR195a-mediated CSF1 inhibition by acting as competing endogenous RNA (ceRNA). miR-195a directly targeted circRNA_28313 and CSF1 3'-UTR, and could form a ceRNA network to regulate RANKL+CSF1induced osteoclast differentiation in BMM cells [95]. circRNA_007438 was upregulated during osteoclastogenesis and targeted miRNA-6338 and miRNA-7028-3p. In contrast, circRNA_005108 was downregulated during osteoclastogenesis and targeted miRNA-6975-3p, miRNA-6516-5p, miRNA-486b-5p, and miRNA-31-3p [17]. Further studies of the relationship between circRNA and osteoclast differentiation will be very useful for the treatment of osteoporosis (Table 3).

\section{Interactions between three miRNAs}

Previous studies showed that miRNAs could lead to gene silencing by binding to mRNAs, and competitive endogenous RNAs could regulate gene expression by competitively binding with miRNA response elements (MREs). Therefore, the ceRNA hypothesis revealed a novel mechanism for inter-RNA interactions [96]. Both the circRNA and lncRNA involved in our review had miRNA binding sites, which acted as miRNA sponges in the cells, and then counteracted the inhibitory effects of miRNAs on their target genes, thereby enhancing the levels of target gene expression. This interaction could form a complex ceRNA network, which plays a major role in various biological processes and disease progression. In osteoporosis, some lncRNAs and all circRNAs affected osteoblast and osteoclast differentiation by acting as miRNA sponges. Examination of this interaction will facilitate analysis of the pathogenesis of osteoporosis, and will be advantageous for the development of novel drugs for treating osteoporosis.

\section{Interactions between IncRNAs and miRNAs}

A number of lncRNAs have been shown to interact with miRNAs during osteoblast differentiation [97]. H19/miR675 was reported to suppress mRNA and protein expression of transforming growth factor- $\beta 1$ (TGF- $\beta 1$ ) and histone deacetylase (HDAC) 4/5. This resulted in inhibition of Smad3 phosphorylation, promoting osteoblast differentiation $[98,99]$. As an lncRNA closely related to osteoporosis, H19 can also regulate the $\mathrm{Wnt} / \beta$-catenin signaling pathway through interactions with miRNAs. miR-141 and miR-22 are negative regulatory factors for osteogenesis and the Wnt/ $\beta$-catenin signaling pathway. lncRNA-H19 acts as a sponge for these two miRNAs, thus negatively regulating their functions and thereby promoting osteoblast proliferation [98, 100]. IncRNA MALAT1 is also a regulator of osteogenic differentiation via two mechanisms. First, lncRNA MALAT1 was reported to regulate osterix overexpression in human MSCs, and then bind to and inhibit miR-143 to promote osteogenic differentiation. Second, lncRNA MALAT1 acts as a sponge for miR-204 and increases the expression of Smad4, which activates and supports the expression of ALP and osteocalcin, thereby promoting bone formation and mineralization [101]. As a molecular sponge for miRNAs, lncRNA MODR binds to miR-454 and mitigates its inhibitory effect on Runx2, thereby promoting bone formation [49]. lncRNA KCNQ1OT1 acts as a ceRNA and interacts directly with miR-214, upregulating the expression of BMP2 and osteogenic genes in bone marrow MSCs [49]. IncRNA NTF3-5 was shown to downregulate miR-93-3p, thereby promoting osteogenic differentiation and bone regeneration [102]. IncRNA POIR competes for miR-182 binding sites resulting in downregulation of its target gene, FOXO1. FOXO1 inhibits the classic Wnt signaling pathway by competing with TCF 4 for $\beta$-catenin, thereby promoting bone formation. In particular, the NF- $k B$ pathway was shown to be abnormally activated during inflammation, thereby increasing the expression level of miR182 and reducing the level of lncRNA POIR, disrupting the balance of the IncRNA-POIR-miR-182 
regulatory network and eventually affecting bone formation [103]. IncRNA Linc-ROR as a sponge for miR138 and miR-145 antagonizes the functions of these two miRNAs (both of which are negative regulators of osteogenesis) and inhibits their shared target, ZEB2, eventually activating Wnt/ $\beta$-catenin signaling pathway, which enhances osteogenesis [104].

Besides promoting effects, this interaction was also shown to inhibit osteoblast differentiation. IncRNA HOTAIR downregulation causes decreases in the expression levels of miR-17-5p and, consequently, of the miR17-5p target, SMAD7. After si-lncRNA HOTAIR, the activity of RUNX2, COL1A1 mRNA expression and ALP would be preeminent [105]. IncRNA MIAT could regulate its binding to target genes by sponging miR-150-5p, and its overexpression inhibits osteogenic differentiation [106]. IncRNA-ORLNC1 acts as a ceRNA for miR-296, reducing the ability of this miRNA to promote osteoblast differentiation by targeting Pten [49]. IncRNA MEG3 regulates the expression of miR-133a-3p, and inhibits osteogenic differentiation of BMSCs obtained from postmenopausal women with osteoporosis [107]. IncRNA TSIX was shown to downregulate the expression of miR30a-5p, and Runx2 expression was suppressed by knockdown of TSIX. Thus, lncRNA TSIX promoted apoptosis of osteoblasts by downregulating miR-30a-5p [108].

Most studies regarding the relationship between lncRNA and miRNA concentrated on the effects on osteogenic differentiation, and there have been few related studies on osteoclasts. One study showed that, during osteoclastogenesis, lncRNA-AK131850 can act as a sponge for miR-93-5p in newborn and mature osteoclasts, increasing vascular endothelial growth factor A (VEGF-A) transcription, expression, and secretion by reducing miR-93-5, thereby promoting vasculogenesis of endothelial progenitor cells [109]. Although this finding was not directly related to osteoporosis, it was closely related to osteoclasts, so it may be useful in developing treatments for osteoporosis.

\section{Interactions between circRNAs and miRNAs}

Previous research had showm that Circular RNAs (circRNAs) serve as competing endogenous RNAs (ceRNAs) and indirectly regulate gene expression through shared microRNAs (miRNAs) [110]. The introduction to circRNAs above mentioned the following pairs of interacting circRNAs and miRNAs: circRNA_0016624 and miR98 [59]; circRNA AFF4 and miR-7223-5p [60]; circRNA CDR1as and miR-7 [61]; and circHIPK3 and miR-124 $[62,63]$. All four pairs of interactions promote osteoblast differentiation. However, the interaction of circIGSF11 and miR-199b-5p inhibits osteoblast differentiation [64]. Moreover, miR-107 inhibits the Wnt/ $\beta$-catenin signaling pathway by downregulating Dkk-1. miR-335 was shown to regulate the osteogenic differentiation of MSCs through the $\mathrm{Wnt} / \beta$-catenin signaling pathway. Analysis of the interaction network showed that circRNA436 is closely related to both miRNAs. Therefore, circRNA436 may affect the Wnt/ $\beta$-catenin signaling pathway as one of the key regulators of osteogenic differentiation [111]. circRNA BANP combined with miRNA-146a, which targets platelet-derived growth factor receptor alpha (PDGFRA) and thus regulates osteogenic differentiation, and circRNA ITCH, exerted this effect by combining with miRNA-34a that targets dual-specificity phosphatase 1 (DUSP1) [112].

circRNAs can affect osteoclast differentiation by binding to multiple miRNAs, e.g., circRNA_007438 binds miRNA-6338 and miRNA-7028-3p. Furthermore, circRNA_005108 was shown to bind to the four miRNAs, miRNA-6975-3p, miRNA-6516-5p, miRNA-486b-5p, and miRNA-31-3p, which contributed to inhibition of osteoclast differentiation [52]. It has been demonstrated that circRNAs are closely related with osteoporosis through their effects as miRNA sponges, but these results were limited. Further studies may facilitate the development of novel treatments for osteoporosis.

\section{Summary and future perspectives}

This review described some RNAs with important roles in the differentiation of osteoblasts and osteoclasts and their molecular mechanisms of action. miRNAs, lncRNAs, and circRNAs act by targeting major genes and signaling pathways related to osteoblast and osteoclast differentiation. Moreover, both lncRNAs and circRNAs can be utilized as miRNA sponges. They play important regulatory roles in various signaling pathways through their interactions with each other, through which they affect cell differentiation. The $\mathrm{Wnt} / \beta$-catenin signaling pathway and its key regulatory molecules, which play important roles in osteoblast differentiation, are targets for multiple regulatory RNAs. The RANK/RANKL/OPG receptor-ligand system is an important element in the differentiation and development of osteoclasts, and these RNAs can act on this system to regulate osteoclast differentiation. The regulation of these two cell types by these three classes of RNAs is essential in the dynamic process of osteoporotic bone metabolism imbalance [52], and so they are intimately related to the development of osteoporosis. Large numbers of miRNAs have been shown to be related to osteoporosis. However, due to the paucity of studies on circRNAs, only a few circRNAs related to osteoblast and osteoclast differentiation have been reported to date.

Osteoporosis has a serious adverse effect on the quality of life of patients and places a major burden on society. 
Therefore, it is necessary to establish the pathogenesis of osteoporosis and develop effective treatments. As molecules that can regulate the differentiation of osteoblasts and osteoclasts through multiple pathways, miRNAs, lncRNAs, and circRNAs can be used as strategic targets or biomarkers with potential application to the diagnosis and treatment of osteoporosis. Furthermore, a better understanding of the regulation of the expression of these RNAs will be instrumental in the development of explicit targeting approaches to treat osteoporosis.

\section{Conclusion}

This review discussed the mechanisms by which miRNAs, IncRNAs, and circRNAs affect osteoporosis, to help in clarifying the shortcomings of current research. Although there are increasing numbers of studies on epigenetics, and current research has also identified a variety of RNAs related to osteoporosis, there is still room for improvement. The discovery of more meaningful RNAs related to osteoporosis, and determination of their interactions, will facilitate further clarification of the pathogenesis of osteoporosis and the development of more efficient drugs and treatment strategies, which will be beneficial to patients, medical practitioners, and to society as a whole.

\begin{abstract}
Abbreviations
miRNAs: microRNAs; IncRNAs: Long non-coding RNAs; circRNAs: Circular RNAs; Hmga2: High mobility group AT-hook 2; Runx2: Runt-related transcription factor 2; MSCs: Mesenchymal stem cells; STAT1: Signal transducer and activator of transcription; DKK1: Dickkopf-1; EGFR: Epidermal growth factor receptor; ERK1: Extracellular signal-regulated kinase 1; HB-EGF: Human ProheparinBinding EGF-like Growth Factor; UTR: 3' Untranslated region; ALP: Alkaline phosphatase; PDLCs: Periodontal ligament cells; hBMSCs: Human bone marrow-derived mesenchymal stem cells; COL1A1: Collagen type I a1; SFRP1: Secreted frizzled-related protein-1; BMD: Bone mineral density; H3K27me3: H3 lysine-27 trimethylation; PDCD4: Programmed cell death protein 4; $\mathrm{HO}-1$ : Heme oxygenase-1; EphA2: Ephrin A receptor 2 precursor; ceRNA: Competing endogenous RNA.
\end{abstract}

\section{Acknowledgements}

Not applicable.

\section{Authors' contributions}

YY and WY designed the manuscript and wrote the part of it. WF wrote the part of manuscript with insights of all other authors. YL and GZ conceptualized and generated Tables 1, 2, 3, Wei Zhichen, Wang Zirui and Wang Shengwang conceptualized and generated Figs. 1, 2, 3, 4. All authors read and approved the final manuscript.

\section{Funding}

No funding.

\section{Availability of data and materials Not applicable.}

Ethics approval and consent to participate Not applicable.

\section{Consent for publication}

Not applicable.

\section{Competing interests}

The authors declare that they have no competing interests.

\section{Author details}

${ }^{1}$ People's Hospital of Guazhou County, Guazhou 736100, Gansu, China.

2 Department of Medical Oncology, Cancer Center, West China Hospital,

Sichuan University, Chengdu 610041, China. ${ }^{3}$ Second Clinical Medical College

of Lanzhou University, Lanzhou 730000, China.

Received: 20 June 2020 Accepted: 7 September 2020

Published online: 16 September 2020

\section{References}

1. Rachner TD, Khosla S, Hofbauer LC. Osteoporosis: now and the future. Lancet. 2011;377(23):1276-87. https://doi.org/10.1016/S0140 $-6736(10) 62349-5$

2. Schuiling KD, Robinia K, Nye R. Osteoporosis update. J Midwifery Womens Health. 2011;56(6):615-27. https://doi.org/10.111 1/j.1542-2011.2011.00135.

3. Feng $\mathrm{Q}$, Zheng $\mathrm{S}$, Zheng J. The emerging role of microRNAs in bone remodeling and its therapeutic implications for osteoporosis. Biosci Rep. 2018;38(3):243-54. https://doi.org/10.1042/BSR20180453.

4. Khan M, Cheung AM, Khan AA. Drug-related adverse events of osteoporosis therapy. Endocrinol Metab Clin North Am. 2017;46(1):181-92. https://doi.org/10.1016/j.ecl.2016.09.009.

5. Lewiecki EM, Binkley N, Bilezikian JP. Treated osteoporosis is still osteoporosis. J Bone Miner Res. 2019;34(4):605-6. https://doi.org/10.1002/ jbmr.3671.

6. Tzouvelekis A, Kaminski N. Epigenetics in idiopathic pulmonary fibrosis. Biochem Cell Biol. 2015;93(2):159-70. https://doi.org/10.1139/ bcb-2014-0126.

7. Yang JJ, Tao H, Deng ZY, Lu C, Li J. Non-coding RNA-mediated epigenetic regulation of liver fibrosis. Metabolism. 2015;64(11):1386-94. https ://doi.org/10.1016/j.metabol.2015.08.004.

8. Rasmussen KD, Jia G, Johansen JV, Pedersen MT, Rapin N, Bagger FO. Loss of TET2 in hematopoietic cells leads to DNA hypermethylation of active enhancers and induction of leukemogenesis. Genes Dev. 2015;29(9):910-22. https://doi.org/10.1101/gad.260174.115.

9. Esteller M. Non-coding RNAs in human disease. Nat Rev Genet. 2011;12(12):861-74. https://doi.org/10.1038/nrg3074.

10. Beermann Julia, Piccoli Maria-Teresa, Viereck Janika, Thum Thomas. Non-coding RNAs in development and disease: background, mechanisms, and therapeutic approaches. Physiol Rev. 2016;96(4):1297-325. https://doi.org/10.1152/physrev.00041.2015.

11. Jin D, Wu X, Yu H, Jiang L, Zhou P, Yao X, et al. Systematic analysis of IncRNAs, mRNAs, circRNAs and miRNAs in patients with postmenopausal osteoporosis. Am J Transl Res. 2018;10(5):1498-510.

12. Bellavia D, Salamanna F, Raimondi L, De Luca A, Carina V, Costa V, et al. Deregulated miRNAs in osteoporosis: effects in bone metastasis. Cell Mol Life Sci. 2019;76(19):3723-44. https://doi.org/10.1007/s00018-01903162-w.

13. Pu M, Chen J, Tao Z, Miao L, Qi X, Wang Y, et al. Regulatory network of miRNA on its target: coordination between transcriptional and post-transcriptional regulation of gene expression. Cell Mol Life Sci. 2018;76(3):441-51. https://doi.org/10.1007/s00018-018-2940-7.

14. Hammond SM. An overview of microRNAs. Adv Drug Deliv Rev. 2015;87:3-14. https://doi.org/10.1016/j.addr.2015.05.001.

15. Shen L, Wu C, Zhang J, Xu H, Liu X, Wu X, et al. Roles and potential applications of IncRNAs in HIV infection. Int J Infect Dis. 2020;92:97-104. https://doi.org/10.1016/j.ijid.2020.01.006.

16. Zhao K, Zhao Q, Guo Z, Chen Z, Hu Y, Su J, et al. Hsa_Circ_0001275: a potential novel diagnostic biomarker for postmenopausal osteoporosis. Cell Physiol Biochem. 2018;46(6):2508-16. https://doi.org/10.1016/j. ijid.2020.01.006

17. Jin, D., Wu, X., Yu, H., Jiang, L., Zhou, P., Yao, X., et al. Systematic analysis of IncRNAs, mRNAs, circRNAs and miRNAs in patients with postmenopausal osteoporosis. Am J Transl Res. 2018; 10(5): 1498-1510, https://www. ncbi.nlm.nih.gov/pmc/articles/PMC5992556/. 
18. Wang F, Wang Y, Zhao Y, Zhan Q, Yu P, Wang J, et al. Sialoglycoprotein isolated from eggs of carassius auratus ameliorates osteoporosis: an effect associated with regulation of the wnt/ $\beta$-catenin pathway in rodents. J Agric Food Chem. 2016;64(14):2875-82. https://doi. org/10.1021/acs.jafc.5b06132.

19. Catalano A, Loddo S, Bellone F, Pecora C, Lasco A, Morabito N, et al. Pulsed electromagnetic fields modulate bone metabolism via RANKL/ $\mathrm{OPG}$ and Wnt/ $\beta$-catenin pathways in women with postmenopausal osteoporosis: a pilot study. Bone. 2018;116(23):42-6. https://doi. org/10.1016/j.bone.2018.07.010.

20. Wang H, Sun Z, Wang Y, Hu Z, Zhou H, Zhang L, et al. miR-33-5p, a novel mechano-sensitive microRNA promotes osteoblast differentiation by targeting Hmga2. Sci Rep. 2016;6(16):23170-8. https://doi.org/10.1038/ srep23170.

21. Gomathi K, Akshaya N, Srinaath N, Moorthi A, Selvamurugan N. Regulation of Runx2 by post-translational modifications in osteoblast differentiation. Life Sci. 2020;245:117-26. https://doi.org/10.1016/j. Ifs.2020.117389.

22. Li J, He X, Wei W, Zhou X. MicroRNA-194 promotes osteoblast differentiation via downregulating STAT1. Biochem Biophys Res Commun. 2015;460(2):482-8. https://doi.org/10.1016/j.bbrc.2015.03.059.

23. Tang X, Lin J, Wang G, Lu J. MicroRNA-433-3p promotes osteoblast differentiation through targeting DKK1 expression. PLOS ONE. 2017;12(6):e0179860. https://doi.org/10.1371/journal.pone.0179860.

24. Yang M, Pan Y, Zhou Y. miR-96 promotes osteogenic differentiation by suppressing HBEGF-EGFR signaling in osteoblastic cells. FEBS Lett. 2014;588(24):4761-8. https://doi.org/10.1016/j.febslet.2014.11.008.

25. Catalano A, Loddo S, Bellone F, Pecora C, Lasco A, Morabito N, et al. Pulsed electromagnetic fields modulate bone metabolism via RANKL/ $\mathrm{OPG}$ and $\mathrm{Wnt} / \beta$-catenin pathways in women with postmenopausal osteoporosis: a pilot study. Bone. 2018;116:42-6. https://doi. org/10.1016/j.bone.2018.07.010.

26. Ma S, Wang DD, Ma CY, Zhang YD. microRNA-96 promotes osteoblast differentiation and bone formation in ankylosing spondylitis mice through activating the Wnt signaling pathway by binding to SOST. $J$ Cell Biochem. 2019;120(9):15429-42. https://doi.org/10.1002/jcb.28810.

27. Feng $Y$, Wan $P$, Yin $L$, Lou X. The inhibition of MicroRNA-139-5p promoted osteoporosis of bone marrow-derived mesenchymal stem cell by targeting Wnt/beta-catenin signaling pathway by NOTCH1. J Microbiol Biotechnol. 2020;30(18):448-58. https://doi.org/10.4014/jmb.

28. Li H, Li T, Fan J, Li T, Fan L, Wang S, et al. miR-216a rescues dexamethasone suppression of osteogenesis, promotes osteoblast differentiation and enhances bone formation, by regulating $\mathrm{c}-\mathrm{Cb}$-mediated PI3K/ AKT pathway. Cell Death Differ. 2015;22(12):1935-45. https://doi. org/10.1038/cdd.2015.99.

29. Xue N, Qi L, Zhang G, Zhang Y. miRNA-125b regulates osteogenic differentiation of periodontal ligament cells through NKIRAS2/NF-KB pathway. Cell Physiol Biochem. 2018;48(4):1771-81. https://doi. org/10.1159/000492350

30. Wang H, Xie Z, Hou T, Li Z, Huang K, Gong J, et al. MiR-125b regulates the osteogenic differentiation of human mesenchymal stem cells by targeting BMPR1b. Cell Physiol Biochem. 2017;41(2):530-42. https://doi. org/10.1159/000457013

31. Huang K, Fu J, Zhou W, Li W, Dong S, Yu S, et al. MicroRNA-125b regulates osteogenic differentiation of mesenchymal stem cells by targeting Cbfß in vitro. Biochimie. 2014;102:47-55. https://doi.org/10.1016/j.bioch i.2014.02.005.

32. Du F, Wu H, Zhou Z, Liu YU. microRNA-375 inhibits osteogenic differentiation by targeting runt-related transcription factor 2. Exp Ther Med. 2015;10(1):207-12. https://doi.org/10.3892/etm.2015.2477.

33. Zhang W, Wu Y, Shiozaki Y, Sugimoto Y, Takigawa T, Tanaka M, et al. miRNA-133a-5p inhibits the expression of osteoblast differentiationassociated markers by targeting the 3' UTR of RUNX2. DNA Cell Biol. 2018:37(3):199-209. https://doi.org/10.1089/dna.2017.3936.

34. Fu HL, Pan HX, Zhao B, Dong BC, Shao L, Fu GS, et al. MicroRNA-100 inhibits bone morphogenetic protein-induced osteoblast differentiation by targeting Smad1. Eur Rev Med Pharmacol Sci. 2016; 20(18): 3911-3919, https://www.europeanreview.org/article/11467.

35. Fan FY, Deng R, Qiu L, Wen Q, Zeng Y, Gao L, et al. MiR-203a-3p. 1 is involved in the regulation of osteogenic differentiation by directly targeting Smad9 in MM-MSCs. Oncol let. 2019;18(6):6339-46. https:// doi.org/10.3892/ol.2019.10994.

36. Li D, Liu J, Guo B, Liang C, Dang L, Lu C, et al. Osteoclast-derived exosomal miR-214-3p inhibits osteoblastic bone formation. Nat Commun. 2016;7(7):10872. https://doi.org/10.1038/ncomms10872.

37. Inoue K, Nakano S, Zhao B. Osteoclastic microRNAs and their translational potential in skeletal diseases. Semin Immunopathol. 2019;41(5):573-82. https://doi.org/10.1007/s00281-019-00761-4.

38. Cao Y, Lv Q, Lv C. MicroRNA-153 suppresses the osteogenic differentiation of human mesenchymal stem cells by targeting bone morphogenetic protein receptor type II. Int J Mol Med. 2015;36(3):760-6. https:// doi.org/10.3892/ijmm.2015.2275.

39. $\mathrm{XuX}$, Zhu L, et al. MiR-124 promotes proliferation and differentiation of osteoblasts via BMP/TGF- $\beta$ signaling pathway. Minerva Endocrinol. 2019;3(12):391-405. https://doi.org/10.23736/S0391-1977.19.03079-7.

40. Qadir AS, Um S, Lee H, Baek K, Seo BM, Lee G, et al. miR-124 negatively regulates osteogenic differentiation and in vivo bone formation of mesenchymal stem cells. J Cell Biochem. 2015;116(5):730-42. https:// doi.org/10.1002/jcb.25026.

41. Tang SL, Huang QH, Wu LG, Liu C, Cai AL. MiR-124 regulates osteoblast differentiation through GSK-3 $\beta$ in ankylosing spondylitis. Eur Rev Med Pharmacol Sci. 2018;22(20):6616-24. https://doi.org/10.26355/eurre v_201810_16136.

42. Zhang $X$, Zhu Y, Zhang C, Liu J, Sun T, Li D, et al. miR-542-3p prevents ovariectomy-induced osteoporosis in rats via targeting SFRP1. J Cell Physiol. 2018;233(9):6798-806. https://doi.org/10.1002/jcp.26430.

43. Kureel J, Dixit M, Tyagi AM, Mansoori MN, Srivastava K, Raghuvanshi A, et al. miR-542-3p suppresses osteoblast cell proliferation and differentiation, targets BMP-7 signaling and inhibits bone formation. Cell Death Dis. 2014;5(6):e1050. https://doi.org/10.1038/cddis.2014.4.

44. Anastasilakis AD, Makras P, Pikilidou M, Tournis S, Makris K, Bisbinas I, et al. Changes of circulating MicroRNAs in response to treatment with teriparatide or denosumab in postmenopausal osteoporosis. J Clin Endocrinol Metab. 2018;103(3):1206-13. https://doi.org/10.1210/ jc.2017-02406.

45. Kocijan R, Weigl M, Skalicky S, Geiger E, Ferguson J, Leinfellner G, et al. MicroRNA levels in bone and blood change during bisphosphonate and teriparatide therapy in an animal model of postmenopausal osteoporosis. Bone. 2020;131(2):115104. https://doi.org/10.1016/j. bone.2019.115104.

46. Huynh NP, Anderson BA, Guilak F, McAlinden A. Emerging roles for long noncoding RNAs in skeletal biology and disease. Connect Tissue Res. 2017;58:116-41. https://doi.org/10.1080/03008207.2016.1194406.

47. Zhu XX, Yan YW, Chen D, Ai CZ, Lu X, Xu SS, et al. Long RNA HoxA-AS3 interacts with $\mathrm{EZH} 2$ to regulate lineage commitment of mesenchymal stem cells. Oncotarget. 2016;7(39):63561-70. https://doi.org/10.18632/ oncotarget.11538.

48. Zhang J, Tao Z, Wang Y. Long non-coding RNA DANCR regulates the proliferation and osteogenic differentiation of human bone-derived marrow mesenchymal stem cells via the p38 MAPK pathway. Int J Mol Med. 2019;41(1):213-9. https://doi.org/10.3892/ijmm.2017.3215.

49. Peng S, Cao L, He S, Zhong Y, Ma H, Zhang Y, et al. An overview of long noncoding rnas involved in bone regeneration from mesenchymal stem cells. Stem Cells Int. 2018;42(28):852-9. https://doi. org/10.1155/2018/8273648

50. Jia Q, Jiang W, Ni L. Down-regulated RNA (IncRNA-ANCR) promotes osteogenic differentiation of periodontal ligament stem cells. Arch Oral Biol. 2015;60(2):234-41. https://doi.org/10.1016/j.archoralbi 0.2014 .10 .007$.

51. Shen JJ, Zhang CH, Chen ZW, Wang ZX, Yang DC, Zhang FL, et al. LnCRNA HOTAIR inhibited osteogenic differentiation of BMSCs by regulating Wnt/ $\beta$-catenin pathway. Eur Rev Med Pharmacol Sci. 2019;23(17):7232-46. https://doi.org/10.26355/eurrev_201909_18826.

52. Yang K, Tian N, Liu H, Tao XZ, Wang MX, Huang W. LncRNAp21 promotes osteogenic differentiation of mesenchymal stem cells in the rat model of osteoporosis by the Wnt/ $/$-catenin signaling pathway. Eur Rev Med Pharmacol Sci. 2019;23(10):4303-9. https://doi.org/10.26355/ eurrev_201905_17935.

53. Li B, Liu J, Zhao J, Ma JX, Jia HB, Zhang Y, et al. LncRNA-H19 modulates wnt/ $\beta$-catenin signaling by targeting $\mathrm{dkk} 4$ in hindlimb unloaded rat. Orthop Surg. 2017;9(3):319-27. https://doi.org/10.1111/os.12321. 
54. Li D, Tian Y, Yin C, Huai Y, Zhao Y, Su P, et al. Silencing of IncRNA AK045490 promotes osteoblast differentiation and bone formation via $\beta$-Catenin/TCF1/Runx2 signaling axis. Int J Mol Sci. 2019;20(24):43-9. https://doi.org/10.3390/ijms20246229.

55. Yin C, Tian Y, Yu Y, Wang H, Wu Z, Huang Z, et al. A novel long noncoding RNA AK016739 inhibits osteoblast differentiation and bone formation. J Cell Physiol. 2019;234(7):11524-36. https://doi.org/10.1002/ jcp.27815.

56. Zhang RF, Liu JW, Yu SP, Sun D, Wang XH, Fu JS, et al. LncRNA UCA1 affects osteoblast proliferation and differentiation by regulating BMP-2 expression. Eur Rev Med Pharmacol Sci. 2019;23(16):6774-82. https:// doi.org/10.26355/eurrev_201908_18715.

57. Li XG, Liu SC, Qiao XF, Kong Y, Liu JG, Peng XM, et al. LncRNA MEG3 promotes proliferation and differentiation of osteoblasts through Wnt/ $\beta$-catenin signaling pathway. Eur Rev Med Pharmacol Sci. 2019;23(11):4521-9. https://doi.org/10.26355/eurrev_201906_18027.

58. Liu Y, Liu C, Zhang A, Yin S, Wang T, Wang Y, et al. Down-regulation of long RNA MEG3 suppresses osteogenic differentiation of periodontal ligament stem cells (PDLSCs) through miR-27a-3p/IGF1 axis in periodontitis. Aging (Albany NY). 2019;11(15):5334-50. https://doi. org/10.18632/aging.102105.

59. Yu L, Liu Y. circRNA_0016624 could sponge miR-98 to regulate BMP2 expression in postmenopausal osteoporosis. Biochem Biophys Res Commun. 2019;516(2):546-50. https://doi.org/10.1016/j. bbrc.2019.06.087.

60. Mi B, Xiong Y, Chen L, Yan C, Endo Y, Liu Y, et al. CircRNA AFF4 promotes osteoblast cells proliferation and inhibits apoptosis via the Mir-7223-5p/ PIK3R1 axis. Aging (Albany NY). 2019;11(24):11988-2001. https://doi. org/10.18632/aging.102524

61. Li X, Zheng Y, Zheng Y, Huang Y, Zhang Y, Jia L. Circular RNA CDR1as regulates osteoblastic differentiation of periodontal ligament stem cells via the miR-7/GDF5/SMAD and p38 MAPK signaling pathway. Stem Cell Res Ther. 2018;9(1):232-8. https://doi.org/10.1186/s13287-018-0976-0.

62. Zhu CY, Yao C, Zhu LQ, She C, Zhou XZ. Dexamethasone-induced cytotoxicity in human osteoblasts is associated with circular RNA HIPK3 downregulation. Biochem Biophys Res Commun. 2019;516(3):645-52. https://doi.org/10.1016/j.bbrc.2019.06.073.

63. Liang J, Shen YC, Zhang XY, Chen C, Zhao H, Hu J. Circular RNA HIPK3 downregulation mediates hydrogen peroxide-induced cytotoxicity in human osteoblasts. Aging (Albany NY). 2020;12(2):1159-70. https://doi. org/10.18632/aging.102674.

64. Zhang M, Jia L, Zheng Y. circRNA expression profiles in human bone marrow stem cells undergoing osteoblast differentiation. Stem Cell Rev Rep. 2019;15(1):126-38. https://doi.org/10.1007/s12015-018-9841-x.

65. Matsuo K, Irie N. Osteoclast-osteoblast communication. Arch Biochem Biophys. 2008;v473(2):201-9. https://doi.org/10.1016/j.abb.2008.03.027.

66. Ducy P. The role of osteocalcin in the endocrine cross-talk between bone remodelling and energy metabolism. Diabetologia. 2011;54(6):1291-7. https://doi.org/10.1007/s00125-011-2155-z.

67. Ren X, Zhou Q, Foulad D, et al. Osteoprotegerin reduces osteoclast resorption activity without affecting osteogenesis on nanoparticulate mineralized collagen scaffolds. Sci Adv. 2019;5(6):eaaw4991. https://doi. org/10.1126/sciadv.aaw4991.

68. Wang S, Xu S, Shi Z, Wu J, Lei S, Wang Y. Progress of research on the relationship between calcitonin gene-related peptide and RANK/RANKL/ OPG system in the bone reconstruction. Zhongguo Xiu Fu Chong Jian Wai Ke Za Zhi. 2019;33(4):511-5. https://doi.org/10.7507/10021892.201811137.

69. Wang S, Liu Z, Wang J, Ji X, Yao Z, Wang X. MiR-21 promotes osteoclastogenesis through activation of PI3K/Akt signaling by targeting Pten in RAW264.7 cells. Mol Med Rep. 2020;13(4):48-56. https://doi. org/10.3892/mmr.2020.10938.

70. Madhyastha R, Madhyastha H, Pengjam Y, Nurrahmah QI, Nakajima Y, Maruyama M. The pivotal role of microRNA-21 in osteoclastogenesis inhibition by anthracycline glycoside aloin. J Nat Med. 2019;73(1):5966. https://doi.org/10.1007/s11418-018-1237-3.

71. Ke K, Sul OJ, Rajasekaran M, Choi HS. MicroRNA-183 increases osteoclastogenesis by repressing heme oxygenase-1. Bone. 2015;2015(81):23746. https://doi.org/10.1016/j.bone.2015.07.006.

72. Mao Z, Zhu Y, Hao W, Chu C, Su H. MicroRNA-155 inhibition upregulates LEPR to inhibit osteoclast activation and bone resorption via activation of AMPK in alendronate-treated osteoporotic mice. IUBMB Life. 2019;71(12):1916-28. https://doi.org/10.1002/iub.2131.

73. Sul OJ, Sung YB, Rajasekaran M, Ke K, Yu R, Back SH, et al. MicroRNA-155 induces autophagy in osteoclasts by targeting transforming growth factor $\beta$-activated kinase 1-binding protein 2 upon lipopolysaccharide stimulation. Bone. 2018;2018(116):279-89. https://doi.org/10.1016/j. bone.2018.08.014.

74. Li J, Xing G, Zhang L, Shang J, Li Y, Li C, et al. Satb1 promotes osteoclastogenesis by recruiting CBP to upregulate miR-223 expression in chronic kidney disease-mineral and bone disorder. Pharmazie. 2017;72(11):680-6. https://doi.org/10.1691/ph.2017.7606.

75. Sun Y, Kuek V, Liu Y, Tickner J, Yuan Y, Chen L, et al. MiR-214 is an important regulator of the musculoskeletal metabolism and disease. J Cell Physiol. 2018;234(1):231-45. https://doi.org/10.1002/jcp.26856.

76. Miller CH, Smith SM, Elguindy M, Zhang T, Xiang JZ, Hu X, et al. RBPJ-regulated miR-182 promotes TNF-a-induced osteoclastogenesis. J Immunol. 2016;196(12):4977-86. https://doi.org/10.4049/jimmu nol.1502044.

77. Kim K, Kim JH, Kim I, Lee J, Seong S, Park YW, et al. MicroRNA-26a regulates RANKL-induced osteoclast formation. Mol Cells. 2015;38(1):75-80. https://doi.org/10.14348/molcells.2015.2241.

78. Mizoguchi F, Murakami Y, Saito T, Miyasaka N, Kohsaka H. MiR-31 controls osteoclast formation and bone resorption by targeting RhoA. Arthritis Res Ther. 2013;15(5):102-10. https://doi.org/10.1186/ar4282.

79. Shi C, Qi J, Huang P, Jiang M, Zhou Q, Zhou H, et al. MicroRNA-17/20a inhibits glucocorticoid-induced osteoclast differentiation and function through targeting RANKL expression in osteoblast cells. Bone. 2014;2014(68):67-75. https://doi.org/10.1016/j.bone.2014.08.004.

80. Chen C, Cheng P, Xie H, Zhou HD, Wu XP, Liao EY, et al. MiR-503 regulates osteoclastogenesis via targeting RANK. J Bone Miner Res. 2014;29(2):338-47. https://doi.org/10.1002/jbmr.2032.

81. Zhou W, Yin H, Wang T, Liu T, Li Z, Yan W, et al. MiR-126-5p regulates osteolysis formation and stromal cell proliferation in giant cell tumor through inhibition of PTHrP. Bone. 2014;2014(66):267-76. https://doi. org/10.1016/j.bone.2014.06.021.

82. Wu Z, Yin H, Liu T, Yan W, Li Z, Chen J, et al. MiR-126-5p regulates osteoclast differentiation and bone resorption in giant cell tumor through inhibition of MMP-13. Biochem Biophys Res Commun. 2014;443(3):9449. https://doi.org/10.1016/j.bbrc.2013.12.075.

83. Dou C, Zhang C, Kang F, Yang X, Jiang H, Bai Y, et al. MiR-7b directly targets DC-STAMP causing suppression of NFATC1 and c-Fos signaling during osteoclast fusion and differentiation. Biochim Biophys Acta. 2014;1839(11):1084-96. https://doi.org/10.1016/j.bbrc.2013.12.075.

84. Yang S, Zhang W, Cai M, Zhang Y, Jin F, Yan S, et al. Suppression of bone resorption by miR-141 in aged rhesus monkeys. J Bone Miner Res. 2018;33(10):1799-812. https://doi.org/10.1002/jbmr.3479.

85. Xie BP, Shi LY, Li JP, Zeng Y, Liu W, Tang SY, et al. Oleanolic acid inhibits RANKL-induced osteoclastogenesis via ER alpha/miR-503/ RANK signaling pathway in RAW264.7 cells. Biomed Pharmacother. 2019;117:109045. https://doi.org/10.1016/j.biopha.2019.109045.

86. Han Y, Liu C, Lei M, Sun S, Zheng W, Niu Y, et al. LncRNA TUG1 was upregulated in osteoporosis and regulates the proliferation and apoptosis of osteoclasts. J Orthop Surg Res. 2019;14(1):416-23. https://doi. org/10.1186/s13018-019-1430-4.

87. Liu C, Cao Z, Bai Y, Dou C, Gong X, Liang M, et al. LncRNA AK077216 promotes RANKL-induced osteoclastogenesis and bone resorption via NFATC1 by inhibition of NIP45. J Cell Physiol. 2019;234(2):1606-17. https ://doi.org/10.1002/jcp.27031.

88. Liu ZZ, Zhang CY, Huang LL, Liu W. Elevated expression of IncRNA SNHG15 in spinal tuberculosis: preliminary results. Eur Rev Med Pharmacol Sci. 2019;23(20):9017-24. https://doi.org/10.26355/eurrev_20191 0_19303.

89. Lee CP, Huang YN, Nithiyanantham S, Huang CM, Ko YC, et al. LncRNAJak3:Jak3 coexpressed pattern regulates monosodium urate crystalinduced osteoclast differentiation through Nfatc1/Ctsk expression. Environ Toxicol. 2019;34(2):179-87. https://doi.org/10.1002/tox.22672.

90. Wang Y, Luo TB, Liu L, Cui ZQ. LnCRNA LINC00311 Promotes the proliferation and differentiation of osteoclasts in osteoporotic rats through the notch signaling pathway by targeting DLL3. Cell Physiol Biochem. 2018;47(6):2291-306. https://doi.org/10.1159/000491539. 
91. Li L, Wang XQ, Liu XT, Guo R, Zhang RD. Integrative analysis reveals key mRNAs and IncRNAs in monocytes of osteoporotic patients. Math Biosci Eng. 2019;16(5):5947-71. https://doi.org/10.3934/mbe.2019298.

92. Chen RS, Zhang XB, Zhu XT, Wang CS. LncRNA Bmncr alleviates the progression of osteoporosis by inhibiting RANML-induced osteoclast differentiation. Eur Rev Med Pharmacol Sci. 2019;23(21):9199-206. https ://doi.org/10.26355/eurrev_201911_19411.

93. Ma X, Guo Z, Gao W, Wang J, Liu Y, Gao F, et al. LncRNA-NEF is downregulated in postmenopausal osteoporosis and is related to course of treatment and recurrence. J Int Med Res. 2019;47(7):3299-306. https:// doi.org/10.1177/0300060519847854.

94. Chang Y, Yu D, Chu W, Liu Z, Li H, Zhai Z. LncRNA expression profiles and the negative regulation of InCRNA-NOMMUT0378352 in osteoclastogenesis. Bone. 2020;130:11507-13. https://doi.org/10.1016/j. bone.2019.115072.

95. Chen X, Ouyang Z, Shen Y, Liu B, Zhang Q, Wan L, et al. CircRNA_28313/ miR-195a/CSF1 axis modulates osteoclast differentiation to affect OVXinduced bone absorption in mice. RNA Biol. 2019;16(9):1249-62. https ://doi.org/10.1080/15476286.2019.1624470.

96. Salmena L, Poliseno L, Tay Y, Kats L, Pandolfi PP. A ceRNA hypothesis: the Rosetta Stone of a hidden RNA language? Cell. 2011;146(5):353-8. https ://doi.org/10.1016/j.cell.2011.07.014.

97. Centofanti F, Santoro M, Marini M, et al. Identification of aberrantlyexpressed long non-coding RNAS in osteoblastic cells from osteoporotic patients. Biomedicines. 2020;8(3):65. https://doi.org/10.3390/biome dicines8030065.

98. Wu QY, Li X, Miao ZN, et al. Long non-coding RNAs: a new regulatory code for osteoporosis. Front Endocrinol (Lausanne). 2018;9:587. https:// doi.org/10.3389/fendo.2018.00587.

99. Huang Y, Zheng Y, Jia L, Li W. Long noncoding RNA H19 promotes osteoblast differentiation via TGF- $\beta 1 /$ Smad3/HDAC signaling pathway by deriving miR-675. Stem Cells. 2015;33(12):3481-92. https://doi. org/10.1002/stem.2225.

100. Liang WC, Fu WM, Wang YB, Sun YX, Xu LL, Wong CW, et al. H19 activates Wnt signaling and promotes osteoblast differentiation by functioning as a competing endogenous RNA. Sci Rep. 2016;6(8):20121-7. https://doi.org/10.1038/srep20121.

101. Wang CG, Liao Z, Xiao H, Liu H, Hu YH, Liao QD, Zhong D. LnCRNA KCNQ10T1 promoted BMP2 expression to regulate osteogenic differentiation by sponging miRNA-214. Exp Mol Pathol. 2019;2019(107):77-84. https://doi.org/10.1016/j.yexmp.2019.01.012.

102. Peng W, Zhu SX, Wang J, Chen LL, Weng JQ, Chen SL. Lnc-NTF3-5 promotes osteogenic differentiation of maxillary sinus membrane stem cells via sponging miR-93-3p. Clin Implant Dent Relat Res. 2018;2018(20):110-21. https://doi.org/10.1111/cid.12553.

103. Wang L, Wu F, Song Y, Li X, Wu Q, Duan Y, et al. Long noncoding RNA related to periodontitis interacts with miR-182 to upregulate osteogenic differentiation in periodontal mesenchymal stem cells of periodontitis patients. Cell Death Dis. 2016;7(8):e2327-32. https://doi. org/10.1038/cddis.2016.125.

104. Feng L, Shi L, Lu YF, Wang B, Tang T, Fu WM, et al. Linc-ROR promotes osteogenic differentiation of mesenchymal stem cells by functioning as a competing endogenous RNA for miR-138 and miR-145. Mol Ther Nucleic Acids. 2018;11(1):345-53. https://doi.org/10.1016/j. omtn.2018.03.004.

105. Wei B, Wei W, Zhao B, Guo X, Liu S. Long RNA HOTAIR inhibits miR-17-5p to regulate osteogenic differentiation and proliferation in non-traumatic osteonecrosis of femoral head. PLoS One. 2017;12(2):e0169097. https://doi.org/10.1371/journal.pone.0169097.

106. Yang L, Li Y, Gong R, Gao M, Feng C, Liu T, et al. The long non-coding rna-orlnc1 regulates bone mass by directing mesenchymal stem cell fate. Mol Ther. 2019;27(2):394-410. https://doi.org/10.1016/j.ymthe .2018.11.019.

107. Wang Q, Li Y, Zhang Y, Ma L, Lin L, Meng J, et al. LncRNA MEG3 inhibited osteogenic differentiation of bone marrow mesenchymal stem cells from postmenopausal osteoporosis by targeting miR-133a-3p. Biomed Pharmacother. 2017;89(12):1178-86. https://doi.org/10.1016/j.bioph a.2017.02.090.

108. Bu Y, Zheng D, Wang L, Liu J. LncRNA TSIX promotes osteoblast apoptosis in particle-induced osteolysis by down-regulating miR-30a-5p. Connect Tissue Res. 2018;59(6):534-41. https://doi.org/10.1080/03008 207.2017.1413362.

109. Quan H, Liang M, Li N, Dou C, Liu C, Bai Y, et al. LnCRNA-AK131850 sponges MiR-93-5p in newborn and mature osteoclasts to enhance the secretion of vascular endothelial growth factor a promoting vasculogenesis of endothelial progenitor cells. Cell Physiol Biochem. 2018;46(1):401-17. https://doi.org/10.1159/000488474.

110. Wang $H$, Zhou $K$, Xiao F, et al. Identification of circRNA-associated ceRNA network in BMSCs of OVX models for postmenopausal osteoporosis. Sci Rep. 2020;10(1):10896. https://doi.org/10.1038/s41598-02067750-8.

111. Huang $X$, Cen $X$, Zhang B, Liao Y, Zhu G, Liu J, et al. Prospect of circular RNA in osteogenesis: a novel orchestrator of signaling pathways. J Cell Physiol. 2019;234(12):21450-9. https://doi.org/10.1002/jcp.28866.

112. Gu X, Li M, Jin Y, Liu D, Wei F. Identification and integrated analysis of differentially expressed IncRNAs and circRNAs reveal the potential ceRNA networks during PDLSC osteogenic differentiation. BMC Genet. 2017;18(1):100-5. https://doi.org/10.1186/s12863-017-0569-4.

\section{Publisher's Note}

Springer Nature remains neutral with regard to jurisdictional claims in published maps and institutional affiliations.
Ready to submit your research? Choose BMC and benefit from:

- fast, convenient online submission

- thorough peer review by experienced researchers in your field

- rapid publication on acceptance

- support for research data, including large and complex data types

- gold Open Access which fosters wider collaboration and increased citations

- maximum visibility for your research: over $100 \mathrm{M}$ website views per year

At BMC, research is always in progress.

Learn more biomedcentral.com/submissions 